\title{
Casas gaming y estadios esports Arquitectura para deportes electrónicos*
}

\author{
Gaming houses and esports stadiums \\ Architecture for electronic sports
}

Jhoan Vidalón** Henry Lazarte***

Universidad Ricardo Palma

Recibido: 29 de octubre de 2019

Aceptado: 30 de noviembre de 2019

\begin{abstract}
RESUMEN
Los deportes electrónicos (e-sports, en inglés) han tenido en los últimos años un enorme impacto internacional y constituyen un fenómeno masivo. El creciente interés de millones de jugadores en todo el mundo motivados por la práctica de deportes electrónicos ha provocado que se construyan edificaciones que faciliten su desarrollo. Unas son las denominadas casas gaming, lugares para entrenar y alojamientos para deportistas. Otros son los estadios esport, que son el equivalente de coliseos en donde se llevan a cabo las competencias. Estos últimos se construyen cada vez en mayor cantidad y con mayores dimensiones, para eventos de enorme concurrencia. En el Perú, si bien existe un gran número de personas que practican esports, aún no se han construido grandes edificaciones para estos deportes lúdicos. Es en este panorama de plataformas tecnológicas, que el trabajo de arquitectos y diseñadores se abre a nuevas posibilidades: producir las ambientaciones virtuales propias de cada videojuego y, por otro lado, proyectar edificios donde los deportistas electrónicos puedan entrenar y competir, de manera profesional.

Palabras clave: Arquitectura, deportes electrónicos, casas gaming, estadios esports, competencia lúdica, profesionalización
\end{abstract}

\begin{abstract}
Electronic sports (e-sports) have had a huge international impact in recent years and form a massive phenomenon. The growing interest of millions of players around the world motivated by the practice of electronic sports has led to the construction of buildings that facilitate their development. Some are the so-called gaming houses, places to train and accommodation for athletes. Others are the esport stadiums, which are the equivalent of coliseums where the competitions are held. The last ones are being built in greater quantity and with greater dimensions each time, for events of enormous attendance. In Peru, although there are a large number of people who practice esports, large buildings have not yet been built for these recreational sports.

It is in this panorama of technological platforms that the work of architects and designers opens up to new possibilities: to produce the virtual settings of each videogame and, on the other hand, to project buildings where electronic athletes can train and compete in a professional manner.
\end{abstract}

Keywords: Architecture, electronic sports, gaming houses, esports stadiums, recreational competition, professionalization.

\footnotetext{
* Antecedentes del documento. El artículo ha sido escrito por Jhoan Vidalón (autor), con la asesoría de Henry Lazarte (coautor). La primera versión se elaboró como parte de la asignatura Pre Tesis, en la Facultad de Arquitectura y Urbanismo, Universidad Ricardo Palma.

** Jhoan Vidalón Ortiz. Egresado de la Facultad de Arquitectura y Urbanismo. Universidad Ricardo Palma (Perú).

*** Henry Lazarte Reátegui. Arquitecto por la Universidad Ricardo Palma (Perú). Master en Hogar Digital, Infraestructura y Servicios por la Universidad Europea de Madrid (España).
} 


\section{Introducción. \\ Tecnología y deporte}

Actualmente, debido al auge del crecimiento tecnológico, lo virtual se va incorporando cada vez más en todas las expresiones de la sociedad, a tal punto que la vida de numerosas personas oscila entre lo físico y lo virtual. $Y$ es que, con la llegada del internet, cada vez más presente en el mundo, se han generado otras formas de interacción social entre las personas. Fruto de estos cambios es que se producen nuevas relaciones económicas, sociales y culturales.

Una de las nuevas manifestaciones se da en el ámbito de los deportes electrónicos, denominados globalmente e-sports, o simplemente esports. Estos deportes son un fenómeno reciente en los países sudamericanos, pero ya tienen un impacto global que se traduce en millones de jugadores en otros países.

Sus inicios, aunque no muy conocidos por los cibernautas, se remontan a la década de 1960, cuando el informático estadounidense Steve Russell creó, en el Instituto Tecnológico de Massachusetts, el programa Spaceward, en uno de los primeros ordenadores DEC PDP-1. (Figura 1) Mediante él, creó un "juego" que para aquel entonces solo se había visto en la ciencia ficción (Cabacas, 2012).

En 1972, en la Universidad de Stanford, se dio la primera competencia de este nuevo juego, a la que se denominó "Olimpiadas intergalácticas”. Esta competencia se llevó a

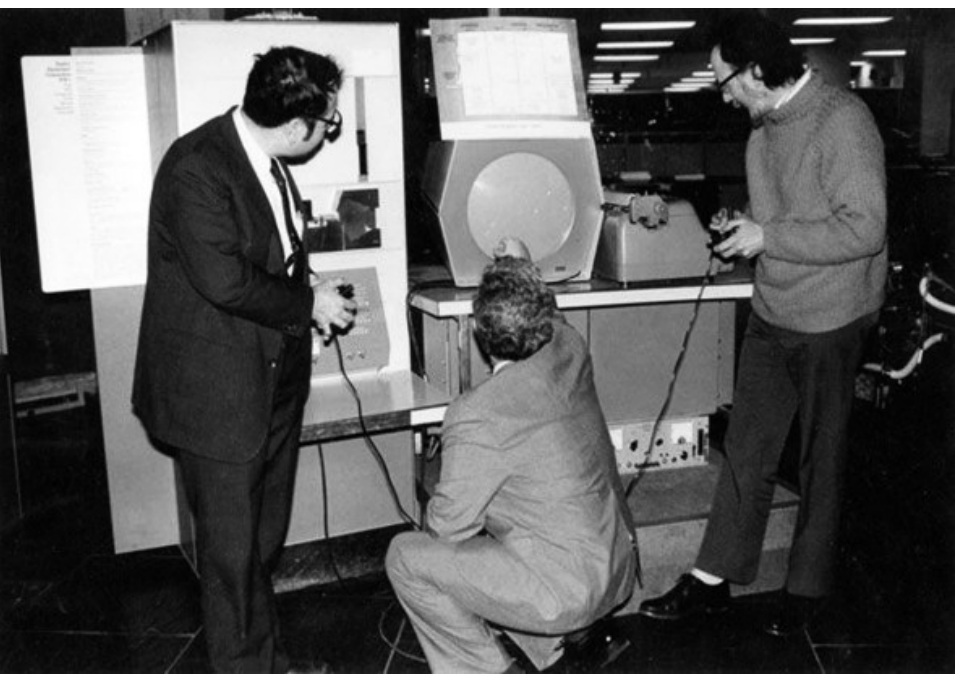

Figura 1: En la primavera de 1961, en el Departamento de Electrónica del MIT fue instalada el PDP-1, uno de los primeros ordenadores fabricados en serie. Fuente: muycomputer.com cabo con múltiples computadoras, cuando las pantallas eran del tamaño de un microondas convencional.

En la década de 1990, los esports se empezaron a difundir con mayor amplitud, ya que en esos años se incrementaron las conexiones a internet, lo cual benefició a quienes practicaban estos juegos, en vista de que ya no tendrían que depender de las conexiones LAN (Local Area Network). Desde entonces pueden interconectarse con muchos más competidores y espectadores a grandes distancias.

Uno de los primeros juegos que se desarrolló con la nueva tecnología fue Netrek, considerado por la revista tecnológica estadounidense Wired como el primer deporte en línea de videojuegos. Paralelamente, se empezaron a desarrollar los primeros torneos esports, como el Nintendo World Championships, al cual siguió el evento Nintendo Powerfest 94 y otros sucesivamente. (Cifaldi, 2016)

Con la ayuda del internet, se generaron sociedades gamer, que empezaron a actuar en países como Canadá, Reino Unido, Australia, etc. De estos, uno de los países que más desarrolló los esports fue Corea del Sur, el cual debe en gran medida su éxito a la KesPA, que, traducido al español, es la Asociación Coreana de Esports, la cual fue promovida por el Ministerio de Cultura, Deporte y Turismo en el año 2000. (Tsang, 2007) Así, con ayuda política, nacieron las primeras ligas profesionales de los esports que, además, con la difusión por canales televisivos, fueron poco a poco tomando mayor importancia en dicho país, hasta el punto de ser más conocidos que los mismos jugadores de fútbol. Gracias a ello, los jugadores profesionales coreanos pudieron desarrollarse y convertirse en una potencia en lo que a deportes electrónicos se refiere. (Gestión, 2019)

Para que los deportistas se desarrollen necesitan espacios o recintos polivalentes (lugar de vida + lugar de trabajo) en constante evolución, en base a nuevos usos tecnológicos, que ahora se conocen como gaming houses. Existen muchos en el mundo y son los lugares donde se reúnen y entrenan los miembros de un equipo profesional. (Figura 2) 
En la actualidad, los deportes electrónicos se están extendiendo de tal manera que empresarios de nivel nacional e internacional invierten millones de dólares en su desarrollo, tanto en infraestructura y marketing, como en investigación y gestión. $Y$ es que, ahora, estos deportes tienen un impacto global que se traduce en millones de jugadores en todos los países que cuentan con acceso a internet, con un interés creciente entre jóvenes de 14 a 30 años.

Firmas de estadísticas económicas, como Newzoo, afirman que en el año 2017 se llegó a tener 385 millones de espectadores en el mundo. Para el año 2019 se calculó una cifra alrededor de los 1000 millones de dólares movidos en relación a este deporte (Collera, 2018) y se espera que los ingresos alcancen los 1100 millones de dólares en 2020. Por otro lado, hubo 443 millones de espectadores en 2019. En 2020, la audiencia total de esports será de 495 millones en todo el mundo, de los cuales 223 millones serán entusiastas, el término utilizado por Newzoo para definir a las personas que consumen contenido de esports más de una vez al mes. Entre todos los países, China destaca en primer lugar y sigue creciendo. De los 2000 millones de personas que han oído hablar de los esports en el mundo, 530 millones residen en China.

\section{Deportes electrónicos y arquitectura}

La arquitectura, como era de esperar, no podía estar ajena a estas actividades. En sus inicios, el juego demandó una nueva organización espacial muy simple. Así, los antiguos ordenadores se dispusieron de manera lineal, a espaldas de los contrincantes. $Y$ es que, como los antiguos ordenadores no estaban destinados a un uso competitivo digital, se buscó adecuarlos a un ambiente de competencia e interacción social virtual. La espacialidad, que en aquel entonces no parecía diferenciarse de ordenar mesas, empezó a tomar rumbo a nuevos paradigmas, que nadie imaginaba en lo que se convertiría algunas décadas más tarde.

Los esports no son juegos de desgaste físico considerable y no necesitan de grandes espacios para practicarse, como los deportes convencionales, llámense fútbol, vóley o básquet. Los jugadores de este tipo de deportes,

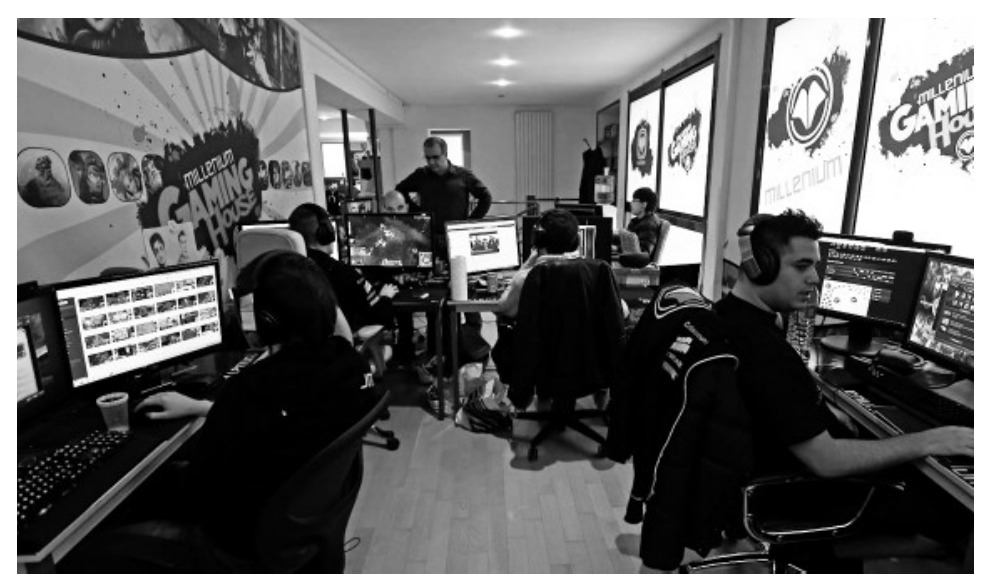

Figura 2: Los jugadores profesionales de Millenium eSport miran las pantallas de sus computadoras mientras juegan dentro de Millenium Gaming House en Marsella. Foto: REUTERS / Jean-Paul Pelissier. Fuente: https://www.firstpost.com/

para poder generar una mejor interacción social que los ayude a tomar decisiones en equipo, propiciaron el surgimiento de las gaming houses. Esta es una nueva tipología de vivienda, prevista para que los compañeros de una agrupación puedan interactuar constantemente entre ellos, así como para relacionarse con mejores jugadores, así vivan a cientos o miles de kilómetros de los centros de competencia.

Por ejemplo, el club estadounidense Evil Geniuses es una organización que participa en diferentes disciplinas, con más de 80 jugadores profesionales. Estos no se agrupan todos en un solo edificio, sino que están en una vivienda con espacios acondicionados para el desarrollo de la actividad, como salas de entrenamiento, gimnasios, saunas e incluso cuartos adicionales para los entrenadores, psicólogos deportivos, cocineros, terapeutas, médicos y, en general, todo aquello que pueda ayudar al mejor desempeño de los jugadores. (Evil Geniuses, 2019) En las gaming houses se contemplan, pues, espacios tales como las salas de entrenamiento y salas de consolas, con capacidad de albergar a cinco jugadores que pueden distribuirse cada uno con sus ordenadores personales. Estos espacios, a su vez, se hallan cercanos a una sala de monitoreo, donde los entrenadores pueden observar los avances del juego y las estrategias a desarrollar; próximas también a salas de reunión, en las que se pueden hacer trámites legales y proyectos. Además, cuentan con los requerimientos de una vivienda con- 
vencional, como habitaciones, que podrían ser grupales o individuales, según el criterio de cada organización, con áreas de entretenimiento, gimnasios y cocinas. Las actividades son supervisadas por un equipo técnico de entrenadores, psicólogos deportivos, directores médicos, fisioterapeutas y mánagers.

Más adelante, surgieron las arenas esports $\mathrm{o}$ arenas gaming. Estas constituyen un fenómeno reciente en el campo del diseño arquitectónico. La arena esport, si bien muestra relaciones directas con los estadios y coliseos convencionales, sus demandas no consisten solo en la visualización de la competencia en sí; a ella se le suman espacios de juego, comercio y relajación, entre otros, en la medida que los participantes pueden transitar, luego del evento principal, por los alrededores de las arenas, en busca de los diferentes servicios para el entretenimiento y goce que se ofrecen a los usuarios.

De modo que las arenas deben contar con una correcta distribución de los ambientes de entrenamiento, preparación y producción. En los espacios de entrenamiento se debe considerar la cantidad estándar de los jugadores a competir, que normalmente en los esports son equipos de cinco personas más el coach. Esto significa que deben contar con Nuevas Tecnologías de la Información y la Comunicación (NTIC) adecuadas para el mantenimiento de computadoras, consolas y sistemas de audio; mientras que la zona de preparación debe ser un salón en el cual los jugadores puedan esperar las siguientes partidas o juegos. $Y$, finalmente, una zona de producción, que cuente con el equipamiento adecuado para transmitir enlaces en línea, ya que actualmente la mayoría de los esports provocan transmisiones a nivel mundial y es su principal forma de difusión. Además, en caso haya periodistas y narradores extranjeros, estos tendrán que contar con espacios de transmisión separados, con planos diversos de los eventos.

Las arenas, pues, se construyen no solo para permitir el desarrollo de los juegos demandados por las comunidades interesadas en las tecnologías de última generación vertidas al entretenimiento y la competencia lúdica, sino también como espacios de socialización. De esta manera, las actividades referidas a juegos digitales se están materializando en interacciones sociales cada vez mayores, en la medida que las arenas deben contar con espacios para recibir a jugadores de diferentes países que necesitan relacionarse. Lo que antes era una actividad privada e individual, ahora se vuelve pública y masiva.

Así, las arenas esports son la base para una industria que alcanza diferentes niveles de desarrollo, según la inversión de la actividad gaming en cada país, pudiendo alcanzar en algunos casos gran difusión internacional.

\section{Tres ejemplos de estadios esports en Norteamérica}

Los edificios más grandes de arquitectura para deportes electrónicos se encuentran en los países asiáticos, aunque en Estados Unidos también se han construido importantes estadios esports y continúan haciéndose.

Uno de los referentes con más inversión en el continente americano es la Arena Esport de Las Vegas, el primer estadio dedicado a los deportes electrónicos en el strip de dicha ciudad y el lugar emblemático de la red global de propiedades de Allied Esports que abarca América del Norte, Europa y China. En este estadio se llevan a cabo distintos torneos en espacios que pueden ser adecuados muy fácilmente, con mucha flexibilidad en los usos. Sin duda, el diseño piramidal acentúa la importancia de los eventos realizados dentro de sus instalaciones y se aprovecha de la misma forma del edificio para difundir la publicidad de los eventos en la fachada. (Figuras 3 y 4 )

En el Estado de Texas se construyó el Esport Stadium de la ciudad de Arlington, con una capacidad de 3000 personas en su área de eventos, una de las construcciones más grandes a nivel mundial. Se construyó bajo el modelo de reutilización adaptativa, donde el objetivo, si bien es con enfoque gaming, puede ser usada para diferentes actividades, ya que con los múltiples espacios de su programación arquitectónica puede realizar eventos simultáneos sin afectar su principal fuente de ingresos, las áreas gaming. A ello se suma el hecho de pensar en un ecosistema que lleve a los usuarios a un uso completo del estadio, con zonas recreativas, salas de conferencias, tiendas, cafés y bares, con- 
virtiéndose en un establecimiento de usos mixtos, que atrae no solo al público aficionado a los juegos, sino que es interactivo con una gran cantidad de personas. (Figuras 5 y 6 )

El Fusion Arena en la ciudad de Filadelfia, en el Estado de Pensilvania, ha sido diseñado por la empresa Comcast Espectocorf. Es un lugar con capacidad de hasta 3500 asientos, con equipamiento exclusivo para deportes electrónicos y zonas de entrenamiento para los equipos que quieran aumentar su competitividad deportiva. El estadio estará ubicado en una zona descentralizada, cerca de otros establecimientos de deportes convencionales, representando un gran impacto puesto que sería la primera arena en Pensilvania dedicada a esta industria. En general, la imagen arquitectónica muestra un diseño de alta tecnología, que si bien cumple el objetivo de representar una infraestructura de gran nivel y sofisticación, cuenta con un esquema simétrico y hasta rígido, puesto que la arena centra su diseño en la zona de espectadores, como se muestra en la publicación de The Cordish Companies. (Figuras 7 y 8 )

\section{Los esports en el Perú}

Los países sudamericanos no tienen el mismo nivel de desarrollo en este tipo de juegos que los asiáticos o los Estados Unidos, salvo, en alguna medida, México, Brasil y Argentina. En general, los principales agentes para su desarrollo se han limitado al uso del internet de banda ancha; con ello, todo se reducía a recibir las innovaciones de sus pares asiáticos, europeos y norteamericanos por medio de campeonatos online, donde los equipos sudamericanos podían participar desde precarias instalaciones, en tanto que algunos jugadores se encontraban en distritos o ciudades lejanas, en vista de que no contaban con un club deportivo formal. Todo esto generó dificultades para iniciarse como deportistas electrónicos.

En el Perú, actualmente, existen varios equipos profesionales, dentro de los cuales un equipo del juego Dota 2 ha podido llegar a representar a nuestro país en dos ocasiones. (Figura 9) Esta agrupación, llamada Infamus Gaming, pudo clasificar al The Internacional uno de los eventos de Valve Corporation con una de las bolsas de premios más grandes del mundo: un monto de 34 millones de dólares repartidos entre los clasificados. (El Internacional, 2019. La República, 2019) El último evento fue desarrollado en Shangai, en el Mercedez Benz Arena diseñado por la ECADI de China, donde 18000 personas fueron a ver a los equipos más importantes del mundo. (Líbero Esport, 2019)

Para los equipos sudamericanos, llegar a estas instancias es similar a clasificar a un mundial de fútbol; como se explicó anteriormente, las agrupaciones con las que se enfrentan cuentan con equipamientos de primer nivel y cumplen con estándares de preparación especializados por cada tipo de juego. Estos pueden ser de un solo jugador o bien de un equipo; las necesidades de los deportistas deben estar adecuadas para garantizar su mejor desempeño en el juego. Frente a ello, la gran mayoría de organizaciones peruanas no garantizan estándares de calidad y mucho menos cuentan con infraestructura adecuada, lo cual constituye una limitación para los jugadores peruanos que, si bien muestran habilidades destacables, no pueden moverse en ámbitos internacionales, salvo alguna que otra excepción.

El hecho cierto es que el Perú aún no cuenta con infraestructura o políticas que incentiven su desarrollo, no obstante que hay muchas personas interesadas en estas actividades. Según la plataforma analítica NEWZOO se estima que actualmente hay 8 millones de personas que han practicado videojuegos. (Business Empresarial, 2019) Esto significaría que casi un tercio de la población peruana está potencialmente interesada en el desarrollo de esta industria. En función a esto, se creó la APDEV - Asociación peruana de deportes electrónicos y videojuegos (APDEV, 2017), institución que tiene como fin promover un diálogo entre los inversionistas y las entidades del Estado para trazar políticas que regulen y den asesorías a personas que tengan interés en desarrollarse en el mundo gamer. Para ello, se han adaptado desde el 2019 algunos centros de entretenimiento con ciertas características tecnológicas. (Figura 10) 


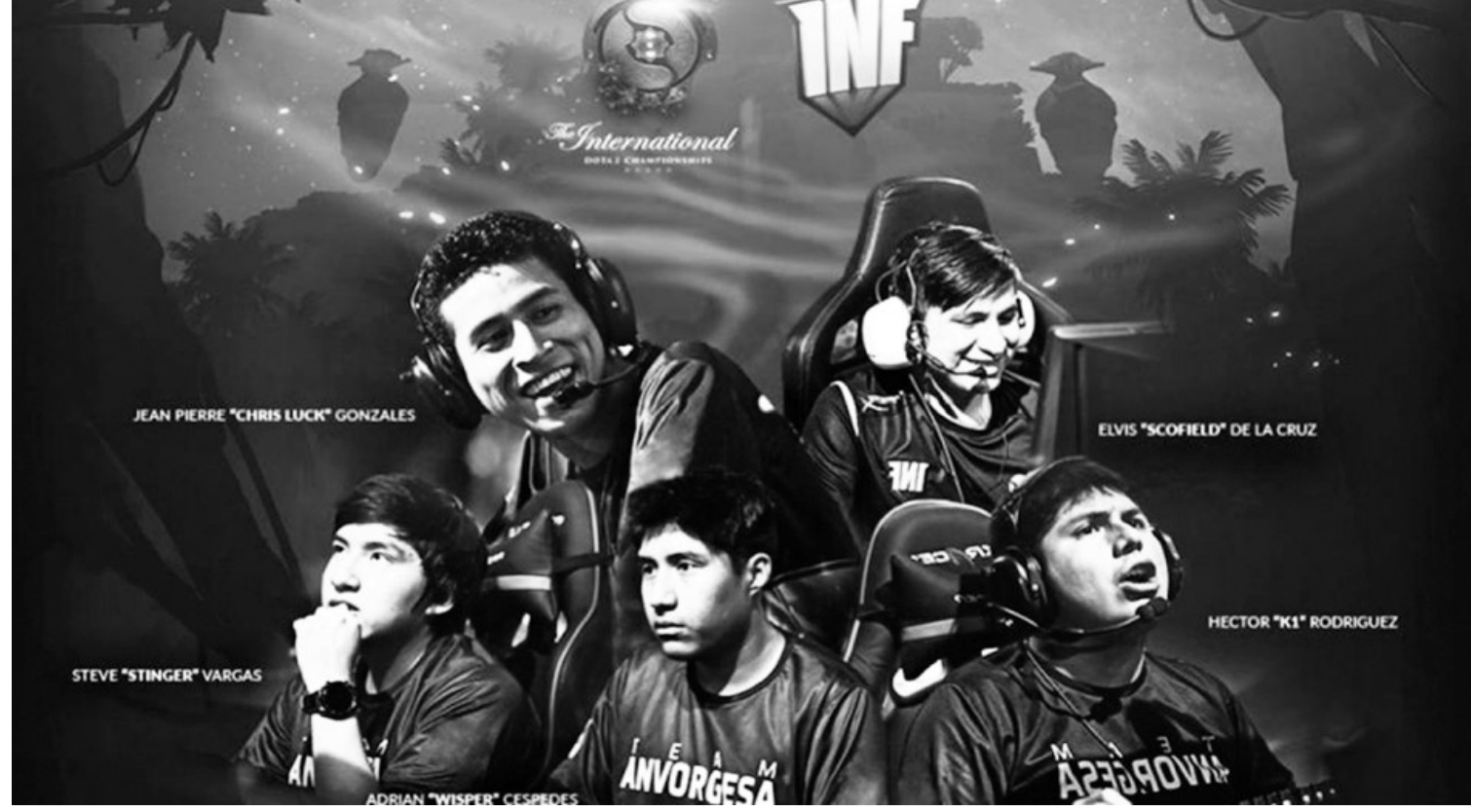

Figura 9. El equipo peruano de Infamous Gaming participó en The International 2019, mundial de Dota 2, en China. Fuente: larepublica.pe

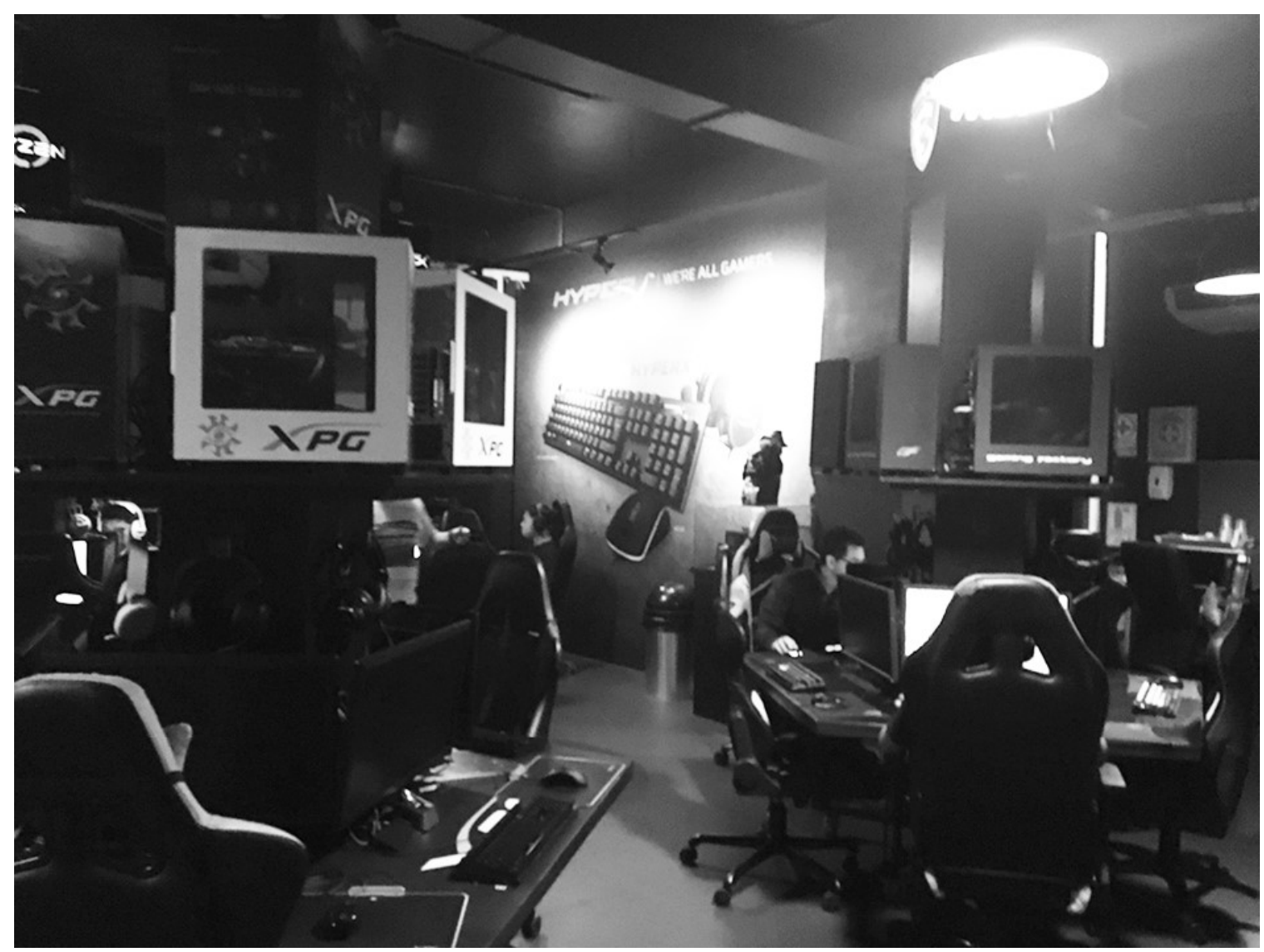

Figura 10. La Empresa Gaming Factory ubicada en un edificio comercial del distrito de Lince, Lima. Foto de Jhoan Vidalón, 2019.

\section{A manera de conclusión}

Los videojuegos son parte innegable de la cultura contemporánea. Si por una parte, la difusión y aceptación de los esports está en marcha a nivel internacional, en el Perú, a pesar de que existe un gran potencial para seguir esta senda, todavía hay muchas limitaciones. Una de estas es la falta de infraestructura.
En este sentido, dadas las proyecciones de las actividades referidas a este tipo de juegos, podría representar en el futuro un campo laboral importante para arquitectos, en la medida que estos nuevos usos necesitarán personas que tengan la capacidad de entender y diseñar espacios que respondan a las nuevas demandas tecnológicas de la era digital. 
Por ejemplo, en el juego La Tierra-Media: sombras de guerra, donde si bien lo principal del juego es la aparición de los soldados luchando con el dragón, estos surgen en un contexto medieval, en el cual la arquitectura, que se muestra al fondo, forma parte de la historia en la cual se desarrolla el juego: detalle necesario para sumergir a los usuarios en un mundo de fantasía que no se daría sin el aporte del contexto arquitectónico. Muchos videojuegos necesitan diseños de espacios virtuales, donde sea posible escapar de la realidad y sumergirse en nuevos mundos.

\section{Referencias bibliográficas}

APDEV (2017). Obtenido de https://apde.org.pe/\#block49

Business Empresarial. (2019). Obtenido de http://www. businessempresarial.com.pe/la-fiebre-de-los-esports-peru-ya-cuenta-con-8-millones-de-gamers/

Cabacas, T. (13 de febrero de 2012). MC. Obtenido de NOTICIAS: https://www.muycomputer.com/2012/02/13/50anos-de-spacewar-el-primer-videojuego/

Cifaldi, F. (14 de julio de 2016). IGN. Obtenido de ASTYANAX: https://www.ign.com/articles/2015/05/13/thestory-of-the-first-nintendo-world-championships

Collera, V. (14 de febrero de 2018). El País. Obtenido de REPORTAJES: https://elpais.com/elpais/2018/02/06/ eps/1517934606_590806.html

EL Internacional. (2019). Obtenido de https://dota2.prizetrac.kr/international2019

Evil Geniuses. (2019). Esto es genios malos. Obtenido de http://www.evilgeniuses.gg/about-us/

Gestión. (12 de marzo de 2019). Tecnología. Obtenido de https://gestion.pe/tecnologia/auge-esports-deportes-electronicos-corea-sur-nndc-260680-noticia/

La República. (14 de agosto de 2019). Obtenido de https://larepublica.pe/videojuegos/2019/08/04/ dota-2-wisper-miembro-de-infamous-gaming-en-the-international-2019-pide-aliento-incondicional-a-peruanos-ti-2019/

Líbero Esport. (19 de agosto de 2019). Obtenido de https://libero.pe/esports/1501769-tig-conoce-estadio-jugara-mundial-dota-2

Tsang, S. (4 de junio de 2007). The Sidney Mornig Herald. Obtenido de: En una tormenta de guerra: https:// www.smh.com.au/technology/in-a-blizzard-of-warfare-20070604-gdqalm.html?page=fullpage

\section{Páginas web consultadas}

El Comercio. (22 de abril de 2019). Obtenido de https://elcomercio.pe/tecnologia/videojuegos/doce-paises-europa-unen-nueva-federacion-europea-esports-espana-mexico-argentina-noticia-628823-noticia/
En este contexto, arquitectos, diseñadores y otros profesionales pueden desenvolverse, con nuevos medios y posibilidades, produciendo ambientaciones virtuales.

Cuando en el Perú se desarrolle la industria de los videojuegos y su práctica profesional, surgirá también la necesidad de diseñar y construir casas gaming y estadios esport, cada vez más grandes y complejos. Es decir, lugares donde los equipos puedan entrenar y donde los fanáticos concurran a ver las competencias de esta enorme manifestación cultural que sigue extendiéndose por el mundo.

Gutiérrez, J. (1 de junio de 2017). El Mundo. Obtenido de https://www.elmundo.es/tecnologia/2017/06/01/ 592fe453468aeb8d7e8b4674.html

Henríquez, C. (17 de abril de 2018). Tarreo. Obtenido de https://www.tarreo.com/noticias/473849/BlueHole-Studios-prepara-infraestructura-para-los-esports-de-PUBG

It Usser. (3 de enero de 2019). Obtenido de https:// almacenamientoit.ituser.es/noticias-y-actuali$\mathrm{dad} / 2019 / 01 /$ las-competiciones-esports-fomentan-la-construccion-de-centros-de-datos

Lordello, V. (7 de octubre de 2017). EXAME. Obtenido de https://exame.abril.com.br/blog/esporte-executivo/nova-arena-de-e-sports-no-brasil-tem-investimento-milionario/

N Economía. (22 de agosto de 2018). Ecommerce News. Obtenido de https://n-economia.com/noticias/elgran-negocio-de-los-esports/

On E-Stadium. (2019). Faca. Obtenido de https://onestadium.com.br/arena/

Webster, A. (7 de septiembre de 2017). THE VERGE. Obtenido de https://www.theverge. com/2017/9/7/16262422/blizzard-esports-arena-overwatch-hearthstone

\section{Links de interés}

http://www.mercedes-benzarena.com/en/AboutUs/ https://elpais.com/elpais/2018/02/06/ eps/1517934606 590806.html

https://elpais.com/cultura/2018/02/16/1 up/1518792125_376873.html

https://www.youtube.com/watch?v=mLOqCYaoQ_g https://www.elmundo.es/tecnologia/2017/06/01/ 592fe453468aeb8d7e8b4674.html

https://www.youtube.com/watch?v=wYDojRdrNiU https://www.youtube.com/watch?v=mLOqCYaoQ_g https://www.youtube.com/watch?v=MfWQLY3ycwo https://www.youtube.com/watch?v=wYDojRdrNiU https://www.youtube.com/watch?v=fiPBI5qzW4M https://www.youtube.com/watch?v=s-FBLAOgBwk 